\title{
Relationship between quality of life and clinical status in asthma: a factor analysis
}

\author{
E.F. Juniper*, M.E. Wisniewski" ${ }^{\#}$ F.M. Cox ${ }^{\oplus}$, A.H. Emmett ${ }^{\#}$, K.E. Nielsen" ${ }^{\#}$, P.M. O’Byrne ${ }^{+}$
}

Relationship between quality of life and clinical status in asthma: a factor analysis. E.F. Juniper, M.E. Wisniewski, F.M. Cox, A.H. Emmett, K.E. Nielsen, P.M. O'Byrne. (C) ERS Journals Ltd 2004.

ABSTRACT: Many studies have shown that correlation between clinical asthma status and asthma-specific quality of life is only weak to moderate. However, this relationship has never been explored to determine whether the weakness is due to noise of measurement or whether quality of life is a distinct component of asthma health status.

With a database from three clinical trials $(n=763)$, factor analysis was used to explore the relationships between quality of life, measured by the Asthma Quality of Life Questionnaire (AQLQ), and conventional measures of asthma clinical status (symptoms, airway calibre and rescue $\beta_{2}$-agonist use).

The analysis revealed that although patients with severe, poorly controlled asthma tend to have worse quality of life than milder, well-controlled patients, overall asthma health status has four components (factors): asthma-specific quality of life; airway calibre; daytime symptoms and daytime $\beta_{2}$-agonist use, and night-time symptoms and night-time $\beta_{2}$-agonist use.

The clean loading of all 21 outcomes onto four distinct and clinically identifiable factors suggests that, although some weakness of correlation between clinical indices and quality of life may be due to noise of measurement, it is mainly attributable to asthma health status being composed of distinct components.

Eur Respir J 2004; 23: 287-291.
*Dept of Clinical Epidemiology and Biostatistics, McMaster University, and ${ }^{+}$Firestone Institute for Respiratory Health and Dept Medicine, McMaster University, Hamilton, Ontario, Canada. ${ }^{\#}$ GlaxoSmithKline, Research Triangle Park, NC, and "Pfizer Inc., New York City, NY, USA.

Correspondence: E. Juniper, 20 Marcuse Fields, Bosham, West Sussex, PO18 8NA, UK.

Fax: 441243573680

E-mail: juniper@qoltech.co.uk

Keywords: Asthma

health status

measurement

quality of life

Received: June 62003

Accepted after revision: November 172003

This study was supported by GlaxoSmithKline R\&D.
Identifying and treating impaired health-related quality of life is now recognised as an important component of asthma management. International guidelines identify that treatments should not only improve asthma clinical status, and thus reduce the risk of exacerbations and possibly airway remodelling, but should also enable patients to feel and function better in their day-to-day lives [1]. Asthma-specific quality of life questionnaires have been developed and validated so that this aspect of patient management can be accurately measured and treatment effectiveness assessed [2-4]. These questionnaires are now used in both clinical trials [5] and clinical practice [6] alongside the more traditional clinical measures of airway status such as airway calibre, symptoms and markers of inflammation.

The rationale for including both clinical and quality of life measures has been based on the observation that correlations between these two measures are only weak to moderate and therefore patient experiences cannot be imputed from the clinical variables [2-4, 7-9]. Correlations between symptoms and asthma-specific quality of life rarely exceed a Pearson correlation coefficient of 0.6 [2-4, 7-9], and correlations between quality of life and airway calibre are usually $<0.2$ and rarely statistically significant [2, 3, 7-9]. Despite the consistency of these observations [2-4, 7-9], it has been argued that these poor correlations arise through imprecision of measurement (both of clinical status and of quality of life).

To determine whether the weakness of association is solely attributable to noise of measurement or whether quality of life is a distinct component of asthma health status, a factor analysis was conducted [10] using a database from three large clinical trials in which asthma-specific quality of life and the conventional measures of clinical asthma were measured in a similar and standardised manner [11-13]. In addition, the analysis has explored whether the conventional clinical outcomes of symptom severity, airway calibre and rescue $\beta_{2}$-agonist use measure a single concept (clinical asthma) or whether they evaluate separate facets of the condition.

Methods

\section{Clinical trials}

Study design. Full reports of the three clinical trials used in this analysis are published elsewhere [11-13]. Briefly, patients with asthma were randomised, in a double-blind manner, to receive either $42 \mu \mathrm{g}$ salmeterol or placebo twice daily by a metered dose inhaler. All patients used rescue salbutamol $90 \mu \mathrm{g}$ when needed. Outcomes were measured at baseline, and after 4, 8 and 12 weeks of treatment. In the primary analysis only data from the placebo groups were used $(n=763)$ so that the outcomes would not be influenced by the trial interventions. All the clinical and quality of life outcomes measured in these studies have been included in the factor analysis.

Study subjects. In all studies, patients were $\geqslant 12$ yrs with inadequately controlled asthma. All patients had prebronchodilator 
forced expiratory volume in one second (FEV1) of 40-80\% predicted with reversibility $\geqslant 15 \% 30 \mathrm{~min}$ after bronchodilator and an average asthma symptom score of $\geqslant 1.0$ on a four-point scale (see below) during the 2 weeks before randomisation.

Study 1 [11]: regular inhaled steroids $(n=254)$; Study 2 [12]: regular non- $\beta_{2}$-agonist medication (inhaled steroids $65 \%$, theophylline $27 \%$ and cromolyn $6 \%)(n=275)$; Study 3 [3]: nocturnal asthma symptoms on six of 14 nights $(67 \%$ inhaled steroids, $24 \%$ theophylline and $9 \%$ short-acting $\beta_{2}$-agonist only) $(n=234)$.

Outcomes. Asthma Quality of Life Questionnaire [2, 7-9]. This 32-item questionnaire measures the functional problems that are most troublesome to adults with asthma. The items are in four domains (symptoms, activity limitations, environmental stimuli and emotional function). Patients recall their experiences during the previous 2 weeks and score each item on a 7-point scale.

Spirometry. Prebronchodilator FEV1, forced vital capacity (FVC) and peak expiratory flow (PEF) were measured at each clinic visit.

Diary. For 2 weeks before each clinic visit, patients recorded the severity of their daytime symptoms each evening (shortness of breath, chest tightness wheeze and physical activity limitation) ( $0=$ none, $3=$ severe). Each morning, they recorded the severity of night-time symptoms and the number of times they had been woken by their asthma ( $0=$ none, $1=$ once, $2=$ two to three times, $3=$ more than three times). Within $15 \mathrm{~min}$ of rising in the morning and at bedtime, patients measured premedication PEF and at the same time recorded the number of puffs of rescue salbutamol used during that night and day respectively.

\section{Statistical analysis}

First, a principal component analysis was conducted to explore the association (correlation) between the 21 outcome measures. Secondly, varimax orthogonal rotation was used to explore whether the 21 items were made up of distinctive groupings (factors). It was decided a priori that the number of factors in the varimax rotation would be based on the number of Eigenvalues $\geqslant 1.0$ in the principal component analysis [14]. In the primary analysis, which examined combined placebo data from all three trials, just the baseline data were used so that each patient would only contribute one set of outcomes. To examine whether the findings were consistent across the different asthma groups, the analysis was repeated in each of the three studies independently. To determine whether the findings were consistent over time, the analysis was repeated in data collected at 4, 8 and 12 weeks (all studies together). Finally, the intervention patients were analysed at $0,4,8$ and 12 weeks to explore whether salmeterol altered the relationships between the outcomes.

\section{Results}

The principal component analysis revealed that fourteen of the 21 items loaded significantly onto the first factor (prerotation) with an Eigenvalue of 6.9 (fig. 1). These results provide evidence that patients with severe asthma tend to be worse in most outcomes than patients with milder asthma.

The first four factors of the principal component analysis had Eigenvalues $\geqslant 1.0$ and together explained $80.8 \%$ of the variance (fig. 1). Therefore, the varimax rotation was based

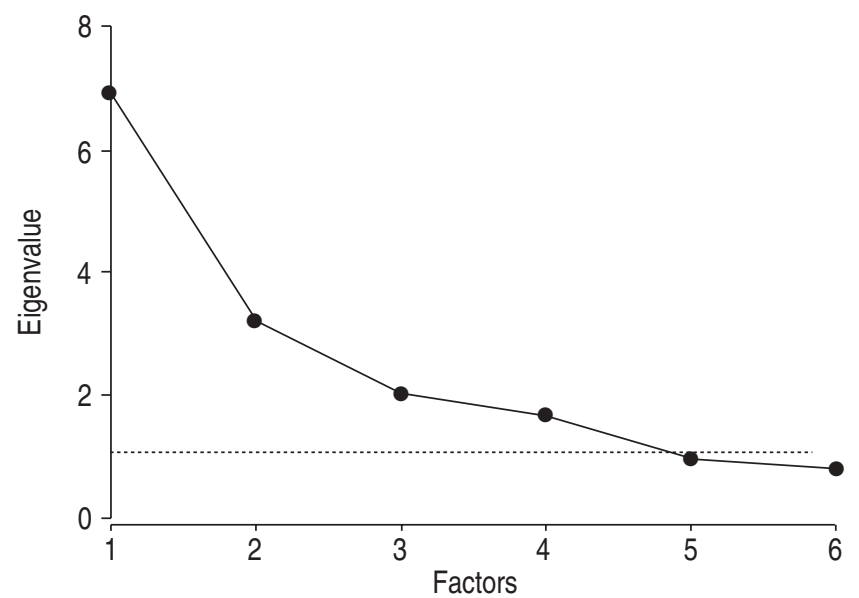

Fig. 1.-Principal component analysis. Four factors had Eigenvalues $\geqslant 1.0(\cdots \cdots)$ and explained $80.8 \%$ of the variance.

on four factors. All but one of the 21 items loaded significantly onto one of the four factors $(\mathrm{p}<0.05)$ (table 1$)$. One factor contains all the quality of life outcomes. A second factor contains the majority of airway calibre measures (PEF and spirometry). Another factor contains all the clinical outcomes associated with night-time asthma (symptoms, nocturnal waking and night-time $\beta_{2}$-agonist use). A fourth factor contains all the outcomes associated with daytime asthma (symptoms, activity limitations and daytime $\beta_{2}$-agonist use).

There were two minor inconsistencies. The one dynamic measure of airway calibre (difference between morning and evening PEF) associated most closely with the fourth factor (daytime asthma). Night-time symptoms correlated most strongly with the other night-time outcomes but also correlated with the daytime outcomes.

Table 1. - Varimax rotated factor pattern (placebo baseline data from all three studies)

\begin{tabular}{|c|c|c|c|c|}
\hline & \multicolumn{4}{|c|}{ Factor } \\
\hline & 1 & 2 & 3 & 4 \\
\hline AQLQ activities & $89^{\#}$ & 11 & -12 & -17 \\
\hline AQLQ environment & $83^{\#}$ & 7 & -3 & -7 \\
\hline AQLQ symptoms & $74^{\#}$ & 6 & -40 & -30 \\
\hline AQLQ emotions & $74^{\#}$ & 5 & -21 & -24 \\
\hline FEV1 & 0 & $92^{\#}$ & -8 & -4 \\
\hline PEF pm & 23 & $84^{\#}$ & -3 & 18 \\
\hline PEF am & 15 & $83^{\#}$ & 0 & -11 \\
\hline FVC & 15 & $80^{\#}$ & -2 & 8 \\
\hline FEF & -19 & $69^{\#}$ & -12 & -16 \\
\hline Night-time waking & -24 & -6 & $84^{\#}$ & 15 \\
\hline Night-time rescue $\beta_{2}$-agonist & -10 & -6 & $75^{\#}$ & 18 \\
\hline Night-time symptoms & -25 & -4 & $51^{\#}$ & $44^{\#}$ \\
\hline Nights without $\beta_{2}$-agonist $\%$ & 13 & 6 & $-77^{\#}$ & -8 \\
\hline Nights without waking \% & 22 & 3 & $-84^{\#}$ & -7 \\
\hline Short of breath daytime & -42 & -7 & 5 & $59^{\#}$ \\
\hline Activity limitation & -48 & -2 & 14 & $56^{\#}$ \\
\hline$\triangle \mathrm{PEF}$ pm-am & 17 & $\overline{5}$ & -5 & $59^{\#}$ \\
\hline Chest tightness daytime & -41 & 0 & 0 & $51^{\#}$ \\
\hline Wheeze daytime & -23 & 6 & 16 & $50^{\#}$ \\
\hline Rescue $\beta_{2}$-agonist daytime & -4 & -10 & 18 & $46^{\#}$ \\
\hline Days without $\beta_{2}$-agonist $\%$ & 4 & 0 & -11 & $-44^{\#}$ \\
\hline
\end{tabular}

For clarity of reading, all values have been multiplied by 100 . AQLQ: Asthma Quality of Life Questionnaire; FEV1: forced expiratory volume in one second; PEF: peak expiratory flow; FVC: forced vital capacity;

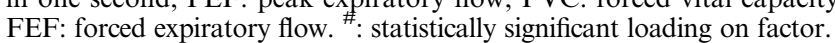


Table 2. - Factor loading across studies and time (varimax rotation) for placebo patients only

\begin{tabular}{|c|c|c|c|c|c|c|c|}
\hline & \multicolumn{3}{|c|}{ Study number (baseline) } & \multicolumn{4}{|c|}{ Week (all studies) } \\
\hline & 1 & 2 & 3 & 0 & 4 & 8 & 12 \\
\hline AQLQ activities & 1 & 1 & 1 & 1 & 1 & 1 & 1 \\
\hline AQLQ environment & 1 & 1 & 1 & 1 & 1 & 1 & 1 \\
\hline AQLQ symptoms & 1,3 & 1 & 1 & 1 & 1 & 1 & 1 \\
\hline AQLQ emotions & 1 & 1 & 1 & 1 & 1 & 1 & 1 \\
\hline FEV $1 \%$ pred & 2 & 2 & 2 & 2 & 2 & 2 & 2 \\
\hline PEF pm & 2 & 2 & 2 & 2 & 2 & 2 & 2 \\
\hline PEF am & 2 & 2 & 2 & 2 & 2 & 2 & 2 \\
\hline $\mathrm{FVC}$ & 2 & 2 & 2 & 2 & 2 & 2 & 2 \\
\hline FEF & 2 & 2 & 2 & 2 & 2 & 2 & 2 \\
\hline Night-time waking & 3 & 3 & 3 & 3 & 3 & 3 & 3 \\
\hline Night-time $\beta_{2}$-agonist use & 3 & 3 & 3 & 3 & 3 & 3 & 3 \\
\hline Night-time symptoms & 3,4 & 3 & 3 & 3,4 & 3,4 & 4,3 & 4,3 \\
\hline Nights without $\beta_{2}$-agonist $\%$ & 3 & 3 & 3 & 3 & 3 & 3 & 3 \\
\hline Nights without waking \% & 3 & 3 & 3 & 3 & 3 & 3 & 3 \\
\hline Short of breath daytime & 4 & 4 & 4 & 4 & 4,1 & 4 & 4 \\
\hline Activity limitation & 4 & 4,1 & 4,1 & 4 & 4,1 & 4 & 4 \\
\hline$\triangle \mathrm{PEF}$ am-pm & 1,4 & 4 & 4 & 4 & 4 & 4 & 4 \\
\hline Chest tightness daytime & 4 & 4 & 4 & 4 & 4 & 4 & 4 \\
\hline Wheeze daytime & 4 & None & None & 4 & 4 & 4 & 4 \\
\hline$\beta_{2}$-agonist use daytime & 4 & 4 & 4 & 4 & 4 & 4 & 4 \\
\hline Days without $\beta_{2}$-agonist $\%$ & 4 & None & None & 4 & 4 & 4 & 4 \\
\hline
\end{tabular}

Factors $(1,2,3$ or 4$)$ onto which each of the 21 items loaded with statistical significance. If an item loaded on two factors, the one onto which it loaded most strongly is shown first. AQLQ: Asthma Quality of Life Questionnaire; FEV1: forced expiratory volume in one second; PEF: peak expiratory flow; FVC: forced vital capacity; FEF: forced expiratory flow.

The repeat analyses in each of the three studies independently and also in the combined data at 4, 8 and 12 weeks yielded results that were very consistent with the primary analysis (table 2). In the salmeterol-treated patients, baseline distribution of outcomes between factors was the same as for the placebo groups (table 3). At 4, 8 and 12 weeks, difference between morning and evening PEF ceased to load onto any of the four factors.

\section{Discussion}

The principal component analysis has confirmed statistically what clinicians already know; namely, that patients with severe asthma have a tendency to have more severe impairment in all outcomes than patients with mild asthma. For instance, a patient who requires high doses of steroid will tend to have worse symptoms, airway calibre, rescue $\beta_{2}$-agonist use and quality of life than a patient who only needs an occasional puff of $\beta_{2}$-agonist.

However, the varimax rotation has revealed a novel finding; namely, that within this overall concept of asthma health status there are four distinct components (factors) that most probably can be labelled "asthma-specific quality of life", "airway calibre", "night-time clinical problems" and "daytime clinical problems". The clean loading of all 21 items onto four distinct and clinically identifiable factors suggests that although some of the weakness of correlation between clinical outcomes and quality of life may be due to noise of measurement, it is mainly due to quality of life being a distinct component of asthma health status.

For those not familiar with factor analysis and its interpretation, considering it in terms of educational assessment might be useful. Results from a general exam usually reveal that overall brighter students have higher scores throughout the paper than not so bright ones (principal component analysis). However, students with special talents will tend to score higher on some questions than others. For instance, a bright mathematics student will tend to do better on the mathematics questions and a linguist will do better on the languages questions. The varimax rotation reveals the specific topics covered by the general exam (e.g. mathematics, languages, fine arts and science). Thus, pooling questions into

Table 3. - Factor loading for salmeterol patients only

\begin{tabular}{lcccc}
\hline & \multicolumn{4}{c}{ Week } \\
\cline { 2 - 5 } & 0 & 4 & 8 & 12 \\
\hline AQLQ activities & 1 & 1 & 1 & 1 \\
AQLQ environment & 1 & 1 & 1 & 1 \\
AQLQ symptoms & 1 & 1 & 1 & 1 \\
AQLQ emotions & 1 & 1 & 1 & 1 \\
FEV1 \% pred & 2 & 2 & 2 & 2 \\
PEF pm & 2 & 2 & 2 & 2 \\
PEF am & 2 & 2 & 2 & 2 \\
FVC & 2 & 2 & 2 & 2 \\
FEF & 2 & 2 & 2 & 2 \\
Night-time waking & 3 & 3 & 3 & 3 \\
Night-time $\beta_{2}$-agonist use & 3 & 3 & 3 & 3 \\
Night-time symptoms & 3,4 & 3,4 & 4,3 & 4,3 \\
Nights without $\beta_{2}$-agonist $\%$ & 3 & 3 & 3 & 3 \\
Nights without waking \% & 3 & 3 & 3 & 3 \\
Short of breath daytime & 4 & 4 & 4 & 4 \\
Activity limitation & 4 & 4 & 4 & 4 \\
$\Delta$ PEF am-pm & 4 & None & None & None \\
Chest tightness daytime & 4 & 4 & 4 & 4 \\
Wheeze daytime & 4 & 4 & 4 & 4 \\
$\beta_{2}$-agonist use daytime & 4 & 4 & 4 & 4 \\
Days without $\beta_{2}$-agonist \% & None & 4 & 4 & 4 \\
\hline
\end{tabular}

Factors (1, 2, 3 or 4$)$ onto which each of the 21 items loaded with statistical significance. If an item loaded on two factors, the one onto which it loaded most strongly is shown first. AQLQ: Asthma Quality of Life Questionnaire; FEV1: forced expiratory volume in one second; PEF: peak expiratory flow; FVC: forced vital capacity; FEF: forced expiratory flow. 
one overall test masks the heterogeneity of the students' abilities and assessing students in only one topic will be a poor indicator of their ability in other topics. Applying this analogy to asthma, it can be seen that, in general, patients with more severe asthma have worse outcomes than patients with mild disease (principal component analysis) but that there are four distinct components to asthma health status (varimax rotation).

The only measurement of dynamic airway calibre (difference in morning and evening PEF) was more closely associated with daytime symptoms than with the actual measures of airway calibre (e.g. FEV1 and PEF). This likely reflects the fact that asthmatic symptoms are more closely associated with variability in lung function over a day than the absolute value made at one particular time. The observation that the activity domain of the AQLQ loaded with the other AQLQ scores and that the diary activity limitation question loaded with the daytime symptoms is not surprising because the diary focussed only on physical activities whereas the AQLQ activity domain also included social, occupational and nonstrenuous activities. Similarly, with symptoms, the AQLQ asks patients about the impact of both respiratory and systemic symptoms on daily experiences whereas the diary asks about the severity of respiratory symptoms.

Although the primary study question addressed the relationship between quality of life and clinical asthma, this analysis has also suggested that clinical asthma may have three components: airway calibre, daytime asthma and nighttime asthma. This observation supports the proposal by KRAFT and MARTIN [15], which others have refuted [16], that nocturnal asthma may be distinct from daytime asthma. In addition, rescue bronchodilator use does not appear to be a distinct factor but is closely associated with either daytime or night-time symptoms depending on when the medication is taken. This finding is not too surprising since rescue medication is usually taken when symptoms are troublesome. Nevertheless, there has been a tendency to analyse $\beta_{2}$-agonist use as a separate outcome pooling day and night-time use into a single value [5]. The present analysis suggests that this may not be the optimum way to examine these data. The present factor analysis concurs with that of BAILEY et al. [17], who showed that airflow impairment and symptoms are separate factors in clinical asthma but their symptom questions did not differentiate between daytime and nocturnal.

In recent years, questionnaires have been developed to measure asthma control [1]. These questionnaires usually include all three of the clinical factors revealed in this analysis and aggregate responses into a single score [18-20]. This is totally appropriate when the outcome "asthma control" has been defined as the composite goal of treatment as identified in the Global Initiative for Asthma (GINA) guidelines [1]. However, analysing the individual components within these questionnaires may provide greater insight into health status and the effects of interventions.

The three studies selected for this analysis were an opportune sample because they were the first studies to yield a large enough sample sizes for a factor analysis with a comprehensive and consistent range of items. Nevertheless, most patients had mild-to-moderate persistent asthma and few were in either the mild-intermittent or severe-persistent range. Although the three studies with different asthma severity samples yielded consistent results (table 2), further studies will be needed to determine whether a similar pattern is present at the extremes of the clinical range of asthma.

It was decided a priori that the primary analysis would include only placebo data in case the intervention had an effect on relationships. If relationships are changed by interventions and these changes can be exposed by factor analysis, it is possible that factor analysis could provide valuable new insights into mechanisms. The secondary analysis showed that the salmeterol-treated patients had exactly the same factor structures at baseline but in all subsequent analyses "difference between morning and evening PEF" ceased to be attached to any of the four factors. Whether this is attributable to the long-acting $\beta_{2}$-agonist (all patients used salbutamol) may be worthy of further investigation. Nevertheless, it does suggest that factor analysis may enable the exploration of changes in relationships.

Although it was specified a priori that the number of factors in the varimax rotation would be based on the number of Eigenvalues $\geqslant 1.0$ in the principal component analysis, exploratory rotations with both three and five factors were nevertheless conducted. The distributions on both three and five factors were much less clinically sensible and strongly suggested that the four factors solution was the most appropriate (results for three and five rotations available on request).

Although the 21 outcomes measured in these clinical trials fell cleanly into four distinct groupings, this does not mean that there are only four factors in asthma. It may be that there are other factors not captured by these 21 items. These might be found among the large number of cellular and biochemical features of asthma (e.g. eosinophils in sputum cells or elevated exhaled nitric oxide) not yet widely used to evaluate asthma status in clinical trials. It may be that these inflammatory markers are associated with other, as yet unidentified, factors. Clearly, additional studies need to be done not only to understand the linking of other outcomes commonly used to evaluate "asthma" but also to explore the mechanisms that produce the four components observed in this study.

This factor analysis has shown clearly that weak correlations between clinical measures and quality of life are primarily due to asthma health status having distinct components. If the poor correlations were due entirely to error of measurement of a single concept, there would have been no separation into such clinically sensible groups. What does this mean clinically? The primary aims of treatment are to prevent mortality, to reduce the probability of future morbidity and to improve patient well-being [21]. In asthma, the conventional clinical outcomes address the first two concerns and quality of life assessment addresses the third. The results of this study have shown very clearly that patient well-being cannot be imputed from clinical outcomes, and that it must be measured and interpreted independently. If the four components are controlled by different mechanisms and interventions affect the components differently, it will be important to know in future which interventions are most effective for which impairment so that clinicians can target individual patient problems.

\footnotetext{
Acknowledgements. The authors would like to thank M. Keech for his valuable input during the preparation of this manuscript.
}

\section{References}

1. National Institutes of Health (National Heart, Lung and Blood Institute). Global Initiative for Asthma; global strategy for asthma management and prevention. Bethesda (MD), National Institutes of Health, 2002 Apr. Publication No. 02-3659.

2. Juniper EF, Guyatt GH, Ferrie PJ, Griffith LE. Measuring quality of life in asthma. Am Rev Respir Dis 1993; 147: 832838 .

3. Marks GB, Dunn SM, Woolcock AJ. A scale for the 
measurement of quality of life in adults with asthma. $J$ Clin Epidemiol 1992; 45: 461-472.

4. Hyland ME, Finnis S, Irvine SH. A scale for assessing quality of life in adult asthma sufferers. $J$ Psychomatic Res 1991; 35: 99-110.

5. Juniper EF, Johnston PR, Borkhoff CM, Guyatt GH, Boulet LP, Haukioja A. Quality of life in asthma clinical trials: comparison of salmeterol and salbutamol. Am J Respir Crit Care Med 1995; 151: 66-70.

6. Jacobs JE, van de Lisdonk EH, Smeele I, van Weel C, Grol RPTM. Management of patients with asthma and COPD: monitoring quality of life and the relationship to subsequent GP interventions. Fam Pract 2001; 18: 574-580.

7. Rutten-van Molken MPMH, Clusters F, Van Doorslaer EKA, et al. Comparison of performance of four instruments in evaluating the effects of salmeterol on asthma quality of life. Eur Respir J 1995; 8: 888-898.

8. Leidy NK, Coughlin C. Psychometric performance of the Asthma Quality of Life Questionnaire in a US sample. Qual Life Res 1998; 7: 127-134.

9. Juniper EF, Norman GR, Cox FM, Roberts JN. Comparison of the standard gamble, rating scale, AQLQ and SF-36 for measuring quality of life in asthma. Eur Respir J 2001; 18: $38-44$.

10. McDonald RP. Factor analysis and related techniques. Hillsdale, NJ, L. Erlbaum, 1985.

11. Kemp JP, Cook DA, Incaudo GA, et al. Salmeterol improves quality of life in patients with asthma requiring inhaled corticosteroids. J Allergy Clin Immunol 1998; 101: 188-195.

12. Busse WW, Casale TB, Murray JJ, Petrocella V, Cox F,
Rickard K. Efficacy, safety and impact on quality of life of salmeterol in patients with moderate persistent asthma. Am J Managed Care 1998; 4: 1579-1587.

13. Lockey RF, DuBuske LM, Friedman B, Petrocella V, Cox F, Rickard K. Nocturnal asthma: effect of salmeterol on quality of life and clinical outcomes. Chest 1999; 115: 666-673.

14. Cattell RB. The Scree test for the number of factors. Multivariate Behav Res 1966; 1: 245-276.

15. Kraft M, Martin RJ. Nocturnal asthma. In: Barnes PJ, Grunstein MM, Leff AR, Woolcock AJ, eds. Asthma. Lippencott-Raven Publishers, Philadelphia, USA, 1997; pp. 2005-2024.

16. Weersink EJ, Postma DS. Nocturnal asthma: not a separate disease entity. Respir Med 1994; 88: 483-491.

17. Bailey WC, Higgins DM, Richards BM, Richards JM. Asthma severity: a factor analytic investigation. $\mathrm{Am} \mathrm{J} \mathrm{Med}$ 1992; 93: 263-269.

18. Juniper EF, O'Byrne PM, Guyatt GH, Ferrie PJ, King DR. Development and validation of a questionnaire to measure asthma control. Eur Respir J 1999; 14: 902-907.

19. Santanello NC, Barber BL, Reiss TF, Friedman BS, Juniper EF, Zhang J. Measurement characteristics of two asthma symptom diary scales for use in clinical trials. Eur Respir J 1997; 10: 646-651.

20. Vollmer WM, Markson LE, O'Connor E, et al. Association of asthma control with health care utilization and quality of life. Am J Respir Crit Care Med 1999; 160: 1647-1652.

21. Guyatt GH, Naylor D, Juniper EF, Heyland D, Cook D and the Evidence-Based Medicine Working Group. Users' guides to the medical literature. IX How to use an article about health-related quality of life. JAMA 1997; 277: 1232-1237. 